response $(\mathrm{CR} / \mathrm{PR})$ following completion of $1 \mathrm{~L}$ chemotherapy according to the label. Eligibility for PARPi treatment will be analysed prospectively by time-to-event by individual country, all countries combined, and in relation to treatment patterns. Finally, between-country variations will be described in relation to $1 \mathrm{~L}$ treatment patterns and national guidelines.

Results In total, eight countries, Austria, Belgium, Denmark, Finland, Israel, Netherlands, Norway, and Portugal, are included in the study (study flow chart; figure 1). Data collection is ongoing and will be finalized by Q1 2021. Inclusion of 120 patients per site will provide precise estimates of local PARPi eligibility (error margin of $4 \%$ ) and sufficient power to detect clinical meaningful differences in PARPi eligibility between any two countries.

Conclusion International real-world OC data is currently scarce. RESPONSE will add to the current knowledge regarding factors influencing eligibility to $1 \mathrm{~L}$ PARPi or PARPi + anti-VEGF maintenance treatment in individual countries, and enable mapping of patient characteristics and key variables in the $1 \mathrm{~L}$ treatment pathway, such as timing and outcome of surgery, including concomitant anti-VEGF treatment.

Disclosures Professor Christian Marth has received funded research from EU, FWF, AstraZeneca and Roche, Honoraria/ Expenses from Roche, Novartis, Amgen, MSD, Pharmamar, AstraZeneca, and Tesaro, and has performed Consulting/Advisory Boards for Roche, Novartis, Amgen, MSD, AstraZeneca, Pfizer, Pharmamar, Cerulean, Vertex, and Tesaro.

Dr Jacob Korach has nothing to disclose.

Dr Kristina Lindemann has acted as Consultant for AstraZeneca, Speaker for AstraZeneca and GSK, and participated in Advisory Boards for AstraZeneca and GSK.

Dr Anne Weng Ekmann-Gade has received research grants for the current trial

Dr E Van Nieuwenhuysen has nothing to disclose.

Dr Heini Lassus has nothing to disclose.

Dr Klaus Kaae Andersen is employed by AstraZeneca.

Jesper Hansen is employed by AstraZeneca.

\section{PROGNOSTIC VALUE OF THE TUMOR INFILTRATING LYMPHOCYTES AND THE NEUTROPHIL-TO-LYMPHOCYTE RATIO IN PATIENTS WITH ADVANCED OVARIAN CANCER}

${ }^{1}$ Beatriz Álvarez-Abril, ${ }^{1}$ Esmeralda García, ${ }^{1}$ Pilar de la Morena, ${ }^{1}$ Alejandra Ivars, ${ }^{1}$ Manuel Sánchez, ${ }^{2}$ Asunción Chaves, ${ }^{3}$ Francisco Pastor, ${ }^{1}$ Gema Marín, ${ }^{1}$ Francisco Ayala, ${ }^{1}$ Elena Garcia Martinez. 'Hospital General Universitario Morales Meseguer; Hematology and Medical Oncology; ${ }^{2}$ Hospital General Universitario Morales Meseguer; Pathology; ${ }^{3}$ Hospital General Universitario Reina Sofía; Pathology

\subsection{6/ijgc-2020-ESG0.132}

Introduction/Background Tumor infiltrating lymphocytes (TIL) and Neutrophil-to-lymphocyte ratio (NLR) have been objectified as independent prognostic factors in different tumours. There is not enough knowledge about the prognostic value of these two factors as a combination. This analysis aims to study the prognostic significance of TIL and NLR in patients with advanced ovarian cancer (OC).

Methodology Observational, single-center and retrospective analysis of a cohort of 135 patients with advanced stage OC treated between 2002 and 2019. Histological samples of ovarian tissue from the surgery of 92 patients were requested, with informed consent, and tissue microarrays (TMA) were constructed. For the TIL study, immunohistochemical staining of the TMA was made and a quantitative analysis was performed through the morphometric analysis of the lymphocytes. Samples were categorized in relation to total area as TIL $0=$ absence; $1=<25 \%$; $2=25-$ $50 \% ; 3=50-75 \% ; 4 \geq 75 \%$. Neutrophils and lymphocytes levels in peripheral blood at the diagnosis were collected to estimate NLR. Survival analysis was performed using Cox regression.

Results Average age 66 years (36-84 years). Median overall survival (OS): 56 months $(0.92-154 \mathrm{~m})$. FIGO stage: $80 \%$ III, 20\% IV. Histology: $87.2 \%$ papillary serous. ECOG: $18.5 \%$ ECOG 2 at diagnosis. Surgery: primary cytoreduction/after neoadjuvant treatment: 59/59 patients. TIL and NLR study: Both variables were not correlated (Spearman's rho: $-0.259, \mathrm{p}=$ 0.106). $75 \%$ of patients had TILCD3 infiltration $<25 \%$. Median $\mathrm{NLR}=3.72$. The univariate analysis showed a higher OS in patients with TILCD3> 25\% (HR 0.448, 95\% CI 0.19 - 1.02;
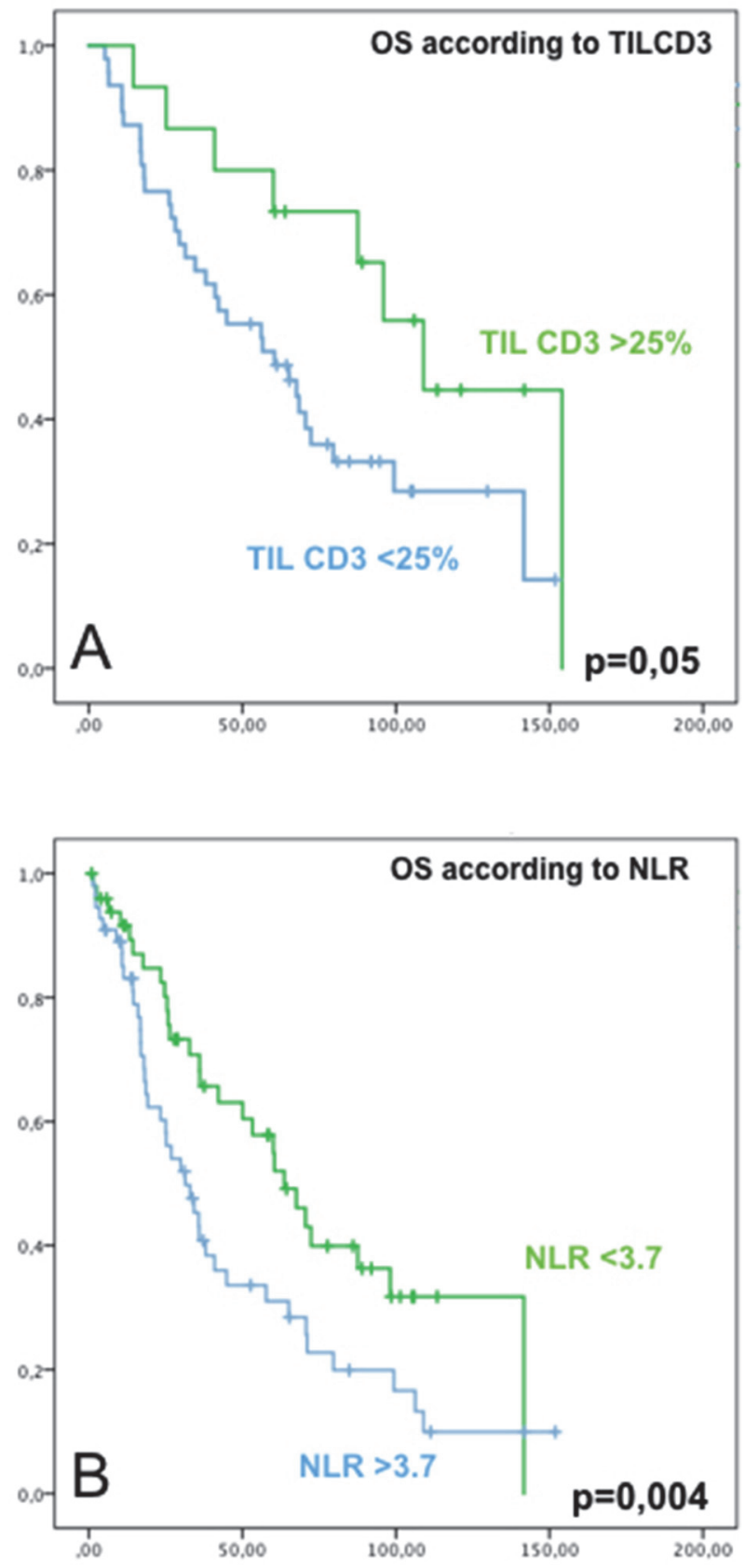

Abstract 413 Figure 1 


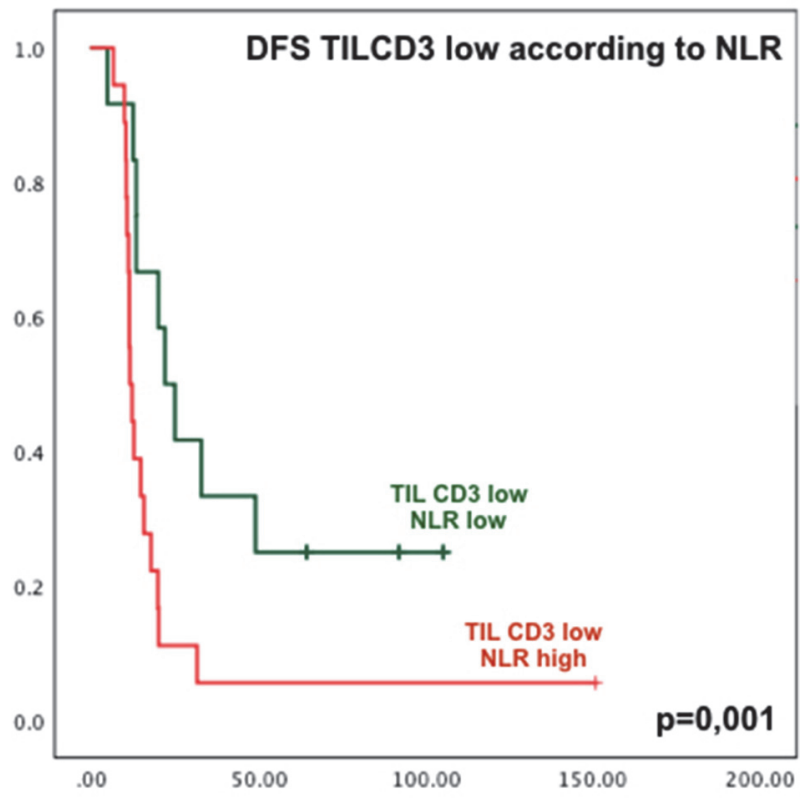

Abstract 413 Figure 2

$\mathrm{p}=0.057)$, and NLR $<3.72(\mathrm{p}=0.004)$ (figure 1$)$. The combined TIL- NLR analysis stratified patients with infiltration by TILCD3 $<25 \%$ into 2 subgroups with clearly different prognoses in terms of disease-free survival (DFS) (median DFS 11.49 vs 21.94 months HR 2.74 95\% CI 1.18 - 6.26; p =0.019) (figure 2). In addition, the combined analysis in the group of patients differentiated four different prognostic subgroups for OS ( $p=$ 0.05). The multivariate analysis including PS and histology showed an independent prognostic value in the variable TILNLR in OS $(p=0.009)$ and DFS ( $p=0.003)$.

Conclusion The combination of TILCD3 and NLR increases their prognostic value in OC. Combination prognostic factor could be very useful for improving immunotherapy strategies in advanced ovarian cancer.

Disclosures B. Álvarez-Abril: None. E. García: None. P. de la Morena: None. A. Ivars: None. M.Sánchez: None. A. Chaves: None. F. Pastor: None. G. Marín: None. F. Ayala de la Peña: None. E. García-Martínez: None.

\section{ELUCIDATING RESISTANCE MECHANISM TO PARP INHIBITORS FOR THE DEVELOPMENT OF NOVEL THERAPEUTIC APPROACHES IN HIGH-GRADE SEROUS OVARIAN CANCER}

\begin{abstract}
${ }^{1}$ Hagen Kulbe, ${ }^{2}$ Wanja Kassuhn, ${ }^{2}$ Frauke Ringel, ${ }^{2}$ Gabriele Welsch, ${ }^{2}$ Peggy Treffkorn, ${ }^{2}$ Eliane Taube, ${ }^{2}$ David Horst, ${ }^{2}$ Jalid Sehouli, ${ }^{2}$ Elena Braicu. ${ }^{1}$ Charité Universitätsmedizin Berlin; Department of Gynecology, Campus Virchow Clinic; ${ }^{2}$ Charité - Universitätsmedizin Berlin, Corporate Member of Freie Universität Berlin, Humboldt-Universität zu Berlin, and Berlin Institute of Health
\end{abstract}

\subsection{6/ijgc-2020-ESG0.133}

Introduction/Background PARP inhibitors (PARPi) have been established as a targeted therapeutic approach not only in patients with high-grade serous ovarian cancer (HGSOC) that have genetic loss of function of BRCA1/2-associated DNA repair. However, treatment efficacy varies and neither BRCA mutation, nor homolog recombination deficiency (HRD) status seem to be optimal predictors. Moreover, mechanisms of treatment resistance are poorly understood and novel approaches are urgently required.

Methodology Here we created gene expression data of HGSOC patients $(n=52)$ before PARPi treatment to elucidate key signaling pathways of resistance to increase their efficacy in combinatorial therapeutic strategies. We performed a comprehensive bioinformatics analysis of the differentially expressed genes between the $25 \%$ extreme responders $(n=26$; 13 each group), including gene set enrichment analysis (GSEA) and causal inference analysis with the CARNIVAL pipeline to elucidate the underlying molecular and regulatory mechanisms governing treatment efficacy and resistance.

Results In accordance with recent publications, we found higher levels of MYC activity in non-responders and deregulation of the $\mathrm{Wnt} / ß$-catenin signaling pathway resulting in PARPi treatment resistance. The pathway enrichment analysis also revealed specific pathways especially PDGFR, FGFR, PI3K/ mTOR and MAPK signaling pathway associated with resistant phenotype. Furthermore, we have identified key kinases, particularly JAK1/2 and SRC that might mediate resistance to PARP inhibition. In addition, differential gene expression analysis revealed folate receptor 1 (FOLR1) to be significantly higher expressed in non-responders $(\operatorname{logFC}=2.66 ; \mathrm{p}<$ 0.0026) with the potential as a serum-based biomarker not only for ovarian cancer, as it correlates closely with CA125, but also PARPi treatment efficacy.

Conclusion In conclusion, these findings define a network of pathways, that are crucial to mediate mechanism of PARPi resistance and identified key signaling kinases as therapeutic targets in ovarian cancer.

Disclosures The authors declare no conflict of interest.

\section{FIRST REAL-WORLD HEMATOLOGIC ADVERSE EVENTS EXPERIENCE WITH NIRAPARIB IN ADVANCED OVARIAN CANCER}

Junjian Wang, Jianqing Zhu. Cancer Hospital of the University of Chinese Academy of Sciences (zhejiang Cancer Hospital)

\subsection{6/ijgc-2020-ESG0.134}

Introduction/Background Niraparib, a poly (ADP-ribose) polymerase (PARP) 1/2 inhibitor, has been approved by Food and Drug Administration (FDA) for $\geq 3$ line recurrence ovarian cancer (OC), the platinum-sensitive recurrence maintenance treatment and new diagnosed maintenance treatment using individual starting dose (ISD, $200 \mathrm{mg}$ daily for body weight $<77 \mathrm{~kg}$ or platelet count $<150,000 / \mu \mathrm{L}$, $300 \mathrm{mg}$ daily for body weight $\geq 77 \mathrm{~kg}$ and $\geq 150,000 / \mu \mathrm{L}$ ). This study aimed to retrospectively assess the incidence of hematologic adverse events (AEs) in real-world Chinese OC patients using ISD niraparib in Zhejiang Cancer Hospital.

Methodology All medical records of OC patients with ISD niraparib in the Zhejiang Cancer Hospital from February 2019 to January 2020 were reviewed. Treatment-emergent hematologic AEs including leukopenia, anemia and thrombocytopenia were collected and analyzed.

Results A total of 43 patients with OC were included in this study. The median body weight was $50.5(33,75) \mathrm{kg} .200$ mg QD was taken as ISD for all patients. Twenty seven $(62.8 \%)$ patients were of BRCA wild-type, 14 (32.6\%) were of BRCA mutants and $2(4.6 \%)$ were unknown. Niraparib 